PROCEEDINGS OF THE AMERICAN MATHEMATICAL SOCIETY

Volume 124, Number 6, June 1996

\title{
ON THE FIXED POINT SETS OF SMOOTH INVOLUTIONS ON THE PRODUCTS OF SPHERES
}

\author{
HUAJIAN YANG
}

(Communicated by Thomas Goodwillie)

\begin{abstract}
In this paper, we have, under some conditions on cohomology, that the fixed point set of a smooth involution on a product of spheres is of constant dimension.
\end{abstract}

\section{INTRODUCTION}

Throughout this paper, we assume $G=Z_{2}$. Let $G$ act smoothly on a smooth closed manifold $M$ with fixed point set $F$. Denote by $M_{G}$ the Borel construction associated with a $G$ action on $M$, and by $p: M_{G} \rightarrow B_{G}=R P^{\infty}$ the fibre bundle with fibre $M$. It is well known that if $F$ is nonempty, then it is a disjoint union of finite number of smooth closed submanifolds of $M$. In this paper, we study the relations between the dimensions of the components of $F$ and the cohomology of $M$ or $M_{G}$. We will prove

Theorem 1.1. Let $M^{n}$ be a smooth closed manifold with a smooth involution $\tau$. Then the fixed point set $F$ is either empty or of constant dimension if one of the following conditions is satisfied:

(i) $H^{*}\left(M_{G} ; Z\right)$ has a generator set $\left\{1, y_{j}\right\}$ as an algebra over $H^{*}\left(R P^{\infty} ; Z\right)$ with $\operatorname{deg}\left(y_{j}\right)$ odd for all possible $j$;

(ii) $\widetilde{H}^{*}\left(M^{n} ; Z\right)$ has no 2-torsions and is algebraically generated by some elements $\left\{x_{i}\right\}$ of odd degrees with $\operatorname{deg}\left(x_{i}\right)+\operatorname{deg}\left(x_{j}\right)>\operatorname{deg}\left(x_{l}\right)$ for $i \neq j$, and $\tau$ induces a trivial $Z_{2}$ action on $\widetilde{H}^{*}\left(M^{n} ; Z\right)$.

Let $R$ be a principal ideal domain. Recall that $M^{n}$ is totally nonhomologous to zero in $M_{G}$ with coefficient in $R$ if the fibre inclusion $j: M^{n} \rightarrow M_{G}$ induces a surjection in cohomology $H^{*}(-; R)([3, \mathrm{p} 373])$. Thus by the Leray-Hirsch theorem [3, Theorem 1.4, p372], the condition (i) of Theorem 1.1 is satisfied if $M^{n}$ is totally nonhomologous to zero in $M_{G}$ with coefficient in $Z$, and $\widetilde{H}^{*}\left(M^{n} ; Z\right)$ has no 2torsions, and is algebraically generated by some elements of odd degrees.

Let $X \sim_{R} Y$ denote two spaces $X$ and $Y$ such that $H^{*}(X ; R)$ and $H^{*}(Y ; R)$ are isomorphic as rings. Denote by $W(M)$ the total Stiefel-Whitney classes of $M$. Note that $W(M)=1$ if $M$ is a product of some spheres. The statement (i) of the next theorem is an immediate corollary of Theorem 1.1.

Received by the editors September 22, 1993 and, in revised form, November 20, 1994.

1991 Mathematics Subject Classification. Primary 57S17.

Key words and phrases. Fixed points, involutions. 
Theorem 1.2. Let $M^{n}$ be a smooth closed manifold with a smooth involution $\tau$. Then $F$ is either empty or of constant dimension, if

(i) $\tau$ induces the trivial $Z_{2}$ action on $H^{*}\left(M^{n} ; Z\right)$, and $M^{n} \sim_{Z} S^{2 n_{1}+1} \times S^{2 n_{2}+1} \times$ $\ldots \times S^{2 n_{k}+1}$ with $2 n_{i}+2 n_{j}>2 n_{l}-1$ whenever $i \neq j\left(\right.$ e.g. $\left.M^{n} \sim_{Z}\left(S^{2 m+1}\right)^{r}\right)$, or

(ii) $\tau$ induces the trivial $Z_{2}$ action on $H^{*}\left(M^{n} ; Z_{2}\right), M \sim Z_{2}\left(S^{l}\right)^{n}$ and $W(M)=1$.

Theorem 1.3. Let $M$ be a smooth closed manifold with $W(M)=1$. Suppose $\tau$ is a smooth involution on $M$ which induces the trivial $Z_{2}$ action on $H^{*}\left(M ; Z_{2}\right)$.

(i) If $M^{2 n} \sim_{Z}\left(S^{2}\right)^{n}$, then $F$ is nonempty and is of constant dimension. Let $k$ be the dimension of $F$. Then $k$ is even and $F$ has at most $2^{n-k / 2}$ components $\left\{F_{i}\right\}$, and for each $F_{i}, H^{*}\left(F_{i} ; Z_{2}\right)$ is algebraically generated by some elements $\left\{b_{i j}\right\}_{1 \leq j \leq n}$ with $b_{i j} \in H^{2}\left(F_{i} ; Z_{2}\right)$ and $b_{i j}^{2}=0$ for all possible $j$. In particular, $H^{*}\left(F_{i} ; Z_{2}\right)$ contains a subring which is isomorphic to $H^{*}\left(\left(S^{2}\right)^{k / 2} ; Z_{2}\right)$.

(ii) Suppose $M \sim Z_{2}\left(S^{1}\right)^{n}$ and $F$ nonempty. Then $F$ is of constant dimension. Let $k$ be the dimension of $F$. Then $F$ has at most $2^{n-k}$ components $\left\{F_{i}\right\}$, and for each $F_{i}, H^{*}\left(F_{i} ; Z_{2}\right)$ is algebraically generated by some elements $\left\{b_{i j}\right\}_{1 \leq j \leq n}$ with $b_{i j} \in H^{1}\left(F_{i} ; Z_{2}\right)$ and $b_{i j}^{2}=0$ for all possible $j$. In particular, $H^{*}\left(F_{i} ; Z_{2}\right)$ contains a subring which is isomorphic to $H^{*}\left(\left(S^{1}\right)^{k} ; Z_{2}\right)$.

We point out, since the statement (i) in [5, Proposition 2.1] (there is a misprint there, $i^{*} c_{k}^{(m)}=c_{k}^{\left(m_{1}\right)}$ should be $i^{*} c_{k}^{(m)}=c_{k}$ ) is true if and only if the smooth involution $\tau$ induces the trivial $Z_{2}$ action on $H^{*}\left(\left(S^{1}\right)^{n} ; Z_{2}\right)$, the main theorem we proved there should be modified as follows.

Theorem. Any smooth involution on $\left(S^{1}\right)^{n}$ with the trivial induced $Z_{2}$ action on $H^{*}\left(\left(S^{1}\right)^{n} ; Z_{2}\right)$ has either empty or constant-dimensional fixed point set $F$.

\section{Proofs of the THEOREMS}

Let $M^{n}$ be a smooth closed manifold with a smooth involution $\tau$. Then $\tau$ induces a $Z_{2}$-equivariant vector bundle structure on the tangent bundle $T\left(M^{n}\right)$ of $M^{n}$. Let $S^{\infty}$ be the infinite-dimensional sphere with a $Z_{2}$ action given by the antipodal involution. Consider the product space $S^{\infty} \times M^{n}$ with the $Z_{2}$ diagonal action. Then projection $S^{\infty} \times M^{n} \rightarrow M^{n}$ is equivariant. Pulling back the $Z_{2}$-equivariant vector bundle $T\left(M^{n}\right)$ by this projection, we obtain a $Z_{2}$-equivariant vector bundle over $S^{\infty} \times M^{n}$, which defines a vector bundle over the Borel space $M_{G}=\left(S^{\infty} \times M^{n}\right) / Z_{2}$ by [1, Proposition 1.6.1, p36]. Denote this vector bundle by $\bar{T}\left(M^{n}\right)$. Similarly, the diagonal action on $S^{m} \times M^{n}$, where the $Z_{2}$ action on $S^{m}$ is given by the antipodal involution, defines a smooth closed manifold $R^{m}(\tau)=\left(S^{m} \times M^{n}\right) / Z_{2}$. Let $p$ denote either projection $\quad R^{m}(\tau) \rightarrow R P(m)$ or $M_{G} \rightarrow R P^{\infty}$. Then $\left(R^{m}(\tau), p, R P(m)\right)$ is a differentiable fibre bundle over $R P(m)$ with fibre $M^{n}$. Consequently, the tangent bundle of $R^{m}(\tau)$ splits and

$$
T\left(R^{m}(\tau)\right) \cong p^{*} T(R P(m)) \oplus \bar{T}_{m}\left(M^{n}\right),
$$

where $\bar{T}_{m}\left(M^{n}\right)$ is called the tangent bundle along the fibres ([2, p482]). Actually, $\bar{T}_{m}\left(M^{n}\right)=i^{*}\left(\bar{T}\left(M^{n}\right)\right.$, where $i: R^{m}(\tau) \rightarrow M_{G}$ is the natural inclusion. Note that the restriction of $\bar{T}_{m}\left(M^{n}\right)$ (or $\bar{T}\left(M^{n}\right)$ ) on a specific fibre is exactly the tangent bundle $T\left(M^{n}\right)$.

Suppose $F \neq \phi$. Given $x \in F$, define $d_{x}$ to be the codimension of the component of $F$ containing $x$, and $I(\tau)$ the set of numbers $d_{x}$. Let $\rho_{x}$ be the section of $p$ associated with $x \in F$. Consider the induced bundle $\eta_{x}^{(m)}=\rho_{x}^{*} \bar{T}_{m}\left(M^{n}\right)$. Observe 
that $d_{x}$ is the number of the eigenvalues $(-1)$ of the local represetation of the group $Z_{2}$ induced by tangent map $d(\tau)$ on the tangent space $T_{x}\left(M^{n}\right)$. This implies the induced bundle $\eta_{x}^{(m)}$ is the Whitney sum of an $\left(n-d_{x}\right)$-dimensional trivial bundle and $d_{x}$ copies of the Hopf bundle. Therefore $W\left(\eta_{x}^{(m)}\right)=(1+a)^{d_{x}}$, where $a \in H^{1}\left(R P(m) ; Z_{2}\right)$ is a generator. Thus

$$
I(\tau)=\left\{d_{x} \mid x \in F, W\left(\eta_{x}^{(m)}\right)=(1+a)^{d_{x}}, 0 \leq d_{x} \leq n\right\}
$$

for every $m>n$.

Remark 2.1. Let $W_{j}(-)$ be the $j$-th Stiefel-Whitney class. Then whenever $m>n$, we have $d_{x}=\max \left\{j \mid W_{j}\left(\eta_{x}^{(m)}\right) \neq 0\right\}=\max \left\{j \mid W_{j}\left(\eta_{x}\right) \neq 0\right\}$, where $\eta_{x}=\rho_{x}^{*} \bar{T}\left(M^{n}\right)$. Let $C(-)$ and $C_{j}(-)$ be the total Chern classes and the $j$-th Chern class respectively. Since $\eta_{x}^{(m)} \otimes C$ is isomorphic to $\rho_{x}^{*}\left(\bar{T}_{m}\left(M^{n}\right) \otimes C\right)$ as complex bundle and

$$
\rho C\left(\eta_{x}^{(m)} \otimes C\right)=\left(W\left(\eta_{x}^{(m)}\right)\right)^{2},
$$

where $\rho$ is the mod 2 reduction homomorphism, we have

$$
\begin{aligned}
d_{x} & =\max \left\{j \mid C_{j}\left(\eta_{x}^{(m)} \otimes C\right) \neq 0\right\} \text { whenever } m>n \\
& =\max \left\{j \mid C_{j}\left(\eta_{x} \otimes C\right) \neq 0\right\} \\
& =\max \left\{j \mid \rho C_{j}\left(\eta_{x} \otimes C\right) \neq 0\right\} .
\end{aligned}
$$

Thus $I(\tau)$ can be computed by using either Stiefel-Whitney or Chern classes.

Let $j$ be the inclusion $M^{n} \rightarrow M_{G}$ or $M^{n} \rightarrow R^{m}(\tau)$. The following theorem shows some relations between $I(\tau)$ and the algebraic structure of $H^{*}\left(M_{G} ; Z\right)$ or $H^{*}\left(M_{G} ; Z_{2}\right)$.

Theorem 2.2. Let $M$ be a smooth closed manifold with a smooth involution $\tau$. Suppose there is a generator set $\left\{c_{i}\right\}$ of $H^{*}\left(M_{G} ; Z\right)$ (resp. $\left.H^{*}\left(M_{G} ; Z_{2}\right)\right)$ as an algebra over $H^{*}\left(R P^{\infty} ; Z\right)$ (resp. $\left.H^{*}\left(R P^{\infty} ; Z_{2}\right)\right)$. If there is an $n_{i}$ such that

$$
\left(c_{i}\right)^{n_{i}} \in p^{*} H^{*}\left(R P^{\infty} ; Z\right)\left(\text { resp. } \in p^{*} H^{*}\left(R P^{\infty} ; Z_{2}\right)\right)
$$

for each $c_{i}$, then $\tau$ has either empty or constant-dimensional fixed point set $F$.

Proof. Suppose $F \neq \phi$. In the case of coefficient $Z_{2}$, consider the homomorphism $\rho_{x}^{*}: H^{*}\left(M_{G} ; Z_{2}\right) \rightarrow H^{*}\left(R P^{\infty} ; Z_{2}\right)$. Then for each $c_{i}$,

$$
\rho_{x}^{*}\left(c_{i}\right)= \begin{cases}0 & \text { if } c_{i}^{n_{i}}=0, \\ a^{m_{i}} & \text { if } c_{i}^{n_{i}} \neq 0,\end{cases}
$$

which is independent of the choices of $x \in F$, where $m_{i}$ is the degree of $c_{i}$ and $a \in$ $H^{1}\left(R P^{\infty} ; Z_{2}\right)$ is a generator. By Remark $2.1, d_{x}=\max \left\{j \mid W_{j}\left(\eta_{x}\right)=\rho_{x}^{*} W_{j}(\bar{T}(M))\right.$ $\neq 0\}$ is independent of the choices of $x \in F$. So $F$ is of constant dimension. In the case of coefficient $Z$, consider the complex bundle $\eta_{x} \otimes C$ and the homomorphism $\rho_{x}^{*}: H^{*}\left(M_{G} ; Z\right) \rightarrow H^{*}\left(R P^{\infty} ; Z\right)$. Just as the preceding case, $\rho_{x}^{*}$ is independent of the choices of $x \in F$. By Remark 2.1 again, $d_{x}=\max \left\{j \mid C_{j}\left(\eta_{x}^{(m)} \otimes C\right)=\right.$ $\left.\rho_{x}^{*} C_{j}(\bar{T}(M) \otimes C) \neq 0\right\}$ is independent of the choices of $x \in F$. So $F$ is also of constant dimension.

Remark 2.3. If $H^{*}\left(M_{G} ; Z_{2}\right)$ (resp. $\left.H^{*}\left(M_{G} ; Z\right)\right)$ has a generator set $\left\{1, x_{1}, x_{2}\right.$, $\left.\ldots, x_{k}\right\}$ as an algebra over $H^{*}\left(R P^{\infty} ; Z_{2}\right)$ (resp. $\left.H^{*}\left(R P^{\infty} ; Z\right)\right)$, then there are at most $2^{k}$ number of different maps $\rho_{x}^{*}$ for $x \in F$. Therefore $F$ has at most $2^{k}$ number of components which are of different dimensions. 
Proof of Theorem 1.1. For (i), $\rho_{x}^{*}\left(y_{j}\right)=0$ for all possible $j$, since $H^{o d d}\left(R P^{\infty} ; Z\right)=$ 0 . Thus the homomorphism $\rho_{x}^{*}$ is independent of the choices of $x \in F$ and (i) follows from Remark 2.1.

For (ii), we consider the spectral sequence $\left\{E_{r}^{p, q}, d_{r}\right\}$ with

$$
E_{2}^{p, q}=H^{p}\left(R P^{\infty} ; H^{q}\left(M^{n} ; Z\right)\right)
$$

([3, p370]), which converges to $H^{*}\left(M_{G} ; Z\right)$. Here the coefficient $H^{q}\left(M^{n} ; Z\right)$ is a local system which becomes constant because of the trivial induced $Z_{2}$ action on $H^{*}\left(M^{n} ; Z\right)$. Let $\left\{x_{i}\right\}$ be the generator set of the $\operatorname{ring} H^{*}\left(M^{n} ; Z_{2}\right)$ with $\operatorname{deg}\left(x_{i}\right)$ odd for all $i$. First note that for each $x_{i}, x_{i}^{2}$ must be of order $\leq 2$, since the degree of $x_{i}$ is odd. Thus we have $x_{i}^{2}=0$, since $H^{q}\left(M^{n} ; Z\right)$ has no 2-torsions. The multiplicative property implies this spectral sequence collapses, since all elements in $E_{2}^{0,2 n_{i}+1}$ and $E_{2}^{2,0}$ are permanent cocycles, where $2 n_{i}+1$ is the degree of some $x_{i}$. Here note that the only possible nontrivial target for the differential $d_{2 r}$ on an element of $E_{2 r}^{0,2 n_{i}+1}$ is in $E_{2 r}^{2 r, 2 n_{i}-2 r+2}$. Since $\operatorname{deg}\left(x_{j}\right)+\operatorname{deg}\left(x_{l}\right)>\operatorname{deg}\left(x_{i}\right)$ for $j \neq l$, we see $E_{2}^{2 r, 2 n_{i}-2 r+2}=0$ for $r \geq 1$. Therefore every element of $E_{2}^{0,2 n_{i}+1}$ is a permanent cocycle. Now the edge homomorphism $H^{q}\left(M_{G} ; Z\right) \rightarrow E_{2}^{o, q} \rightarrow H^{q}\left(M^{n} ; Z\right)$, which is precisely the $j^{*}: H^{*}\left(M_{G} ; Z\right) \rightarrow H^{*}\left(M^{n} ; Z\right)([3, \mathrm{p} 374])$, is surjective, we see that $H^{*}\left(M_{G} ; Z\right)$ is an algebra over $H^{*}\left(R P^{\infty} ; Z\right)$ with generator set $\left\{1, y_{i}\right\}$ and $\operatorname{deg}\left(y_{i}\right)$ odd for all $i$, and (ii) follows just as (i).

Proof of Theorem 1.2. We only need to prove (ii). Consider the spectral sequence which converges to $H^{*}\left(M_{G} ; Z_{2}\right)$ with $E_{2}^{p, q}=H^{p}\left(R P^{\infty} ; H^{q}\left(M ; Z_{2}\right)\right)$. Here the local coefficient system $H^{*}\left(M ; Z_{2}\right)$ again becomes constant because of the trivial induced $Z_{2}$ action on $H^{*}\left(M ; Z_{2}\right)$. By the multiplicative property, this spectral sequence collapses since all elements of $E_{2}^{0, l}$ and $E_{2}^{1,0}$ are permanent cocycles. By [3, Theorem 1.6, p374], $M$ is totally nonhomologous to zero in $M_{G}$ and hence in $R^{m}(\tau)$ with coefficeint in $Z_{2}$ for any $m \geq 0$. Thus $H^{*}\left(R^{m}(\tau) ; Z_{2}\right)$ is a free $H^{*}\left(R P(m) ; Z_{2}\right)$ module with a module basis $\left\{x_{i}\right\}$, where $x_{i}$ runs through all the possible products $\left(c_{1}\right)^{\epsilon_{1}}\left(c_{2}\right)^{\epsilon_{2}} \ldots\left(c_{n}\right)^{\epsilon_{n}}$. Here $\epsilon_{i}=0$ or 1 , and $\left\{c_{1}, c_{2}, \ldots, c_{n}\right\}$ are the elements of $H^{l}\left(M_{G} ; Z_{2}\right)$ such that $\left\{j^{*}\left(c_{1}\right), \ldots, j^{*}\left(c_{k}\right)\right\}$ make up a basis of the $Z_{2}$ vector space $H^{l}\left(M ; Z_{2}\right)$.

Let $V_{i}$ be the $i$-th Wu class of the tangent bundle $T\left(R^{m}(\tau)\right)([4, \mathrm{p} 132])$. If $V_{i} \in p^{*} H^{i}\left(R P(m) ; Z_{2}\right)$ for all $i$, then $W_{k} \in p^{*} H^{k}\left(R P(m) ; Z_{2}\right)$ for all $k$ by the formula

$$
W_{k}=\sum_{i+j=k} S q^{i}\left(V_{j}\right)
$$

where $W_{k}$ is the $k$-th Stiefel-Whitney class of $T\left(R^{m}(\tau)\right)$. This implies $W_{k}\left(\bar{T}_{m}(M)\right)$ $\in p^{*} H^{k}\left(R P(m) ; Z_{2}\right)$ for all $k$ by the facts

$$
W\left(T\left(R^{m}(\tau)\right)\right)=p^{*} W(T(R P(m))) W\left(\bar{T}_{m}(M)\right)
$$

and $W(T(R P(m)))=(1+a)^{m+1}$, where $a \in H^{1}\left(R P(m) ; Z_{2}\right)$ is a generator. Therefore by Remark 2.1, $F$ must be of constant dimension if not empty.

Now suppose $V_{k}$ contains a nontrivial summand of the form $a^{n^{\prime}} c_{i_{1}} c_{i_{2}} \ldots c_{i_{j}}, j \geq 1$. Then we claim $j<n$. To see this, we notice $j^{*} W\left(\bar{T}_{m}(M)\right)=W(M)=1$ and that $\bar{T}_{m}(M)$ is $(n l)$-dimensional. Thus there is no such summands as $a^{n^{\prime}} c_{1} c_{2} \ldots c_{n}$ in $W\left(\bar{T}_{m}(M)\right)$ neither in $W\left(T\left(R^{m}(\tau)\right)\right)$. By using the formula (1), we can write $V_{k}$ 
as the sum

$$
V_{k}=\sum S q^{j_{1}} S q^{j_{2}} \ldots S q^{j_{t}}\left(W_{j}\right), \quad j_{1}+j_{2}+\ldots+j_{t}+j=k .
$$

Since for all $i,\left(c_{i}\right)^{2}=\sum b_{j} c_{j}+b_{0}$, where $b_{0}$ and $b_{j}$ are elements in $p^{*} H^{*}\left(R P(m) ; Z_{2}\right)$, we have

$$
S q^{m^{\prime}}\left(c_{i}\right)=\sum b_{j}^{\prime} c_{j}+b_{0}^{\prime}
$$

for some elements $b_{j}^{\prime}$ and $b_{0}^{\prime}$ in $p^{*} H^{*}\left(R P(m) ; Z_{2}\right)$. Then by $(2)$, there is no such term $a^{n^{\prime}} c_{1} c_{2} \ldots c_{n}$ in $S q^{j_{1}} S q^{j_{2}} \ldots S q^{j_{t}}\left(W_{j}\right)$ neither in $V_{k}$. Therefore, we may assume $V_{k}$ contains a nontrivial summand $a^{n^{\prime}} c_{1} c_{2} \ldots c_{j}, \quad 0<j<n$. Then by the definition of Wu class ([4]),

$$
\begin{aligned}
1 & =\left\langle V_{k}\left(a^{m-n^{\prime}} c_{j+1} c_{j+2} \ldots c_{n}\right), \sigma\right\rangle \\
& =\left\langle S q^{k}\left(a^{m-n^{\prime}} c_{j+1} c_{j+2} \ldots c_{n}\right), \sigma\right\rangle \\
& =0
\end{aligned}
$$

since $S q^{k}\left(a^{m-n^{\prime}} c_{j+1} c_{j+2} \ldots c_{n}\right)$ must be zero by the formula (2). Here $\sigma \in$ $H_{m+n l}\left(R^{m}(\tau) ; Z_{2}\right)$ is the homology fundamental class of the closed manifold $R^{m}(\tau)$. This contradiction shows $V_{k} \in p^{*} H^{*}\left(R P^{\infty} ; Z_{2}\right)$ for all $k$, and (ii) follows.

Proposition 2.4. Let $M$ be a smooth closed manifold with a smooth involution $\tau$ which induces the trivial $Z_{2}$ action on $H^{*}\left(M ; Z_{2}\right)$. Suppose $M \sim_{Z_{2}}\left(S^{2}\right)^{n}$ and $W(M)=1$. Let $F \neq \phi$ and $k$ be the constant dimension of $F$. If $S q^{1}(x) \in$ $p^{*} H^{*}\left(R P^{\infty} ; Z_{2}\right)$ for all $x \in H^{*}\left(M_{G} ; Z_{2}\right)$, then $k$ is even and $F$ has at most $2^{n-k / 2}$ components $\left\{F_{i}\right\}$, and for each $F_{i}, H^{*}\left(F_{i} ; Z_{2}\right)$ is algebraically generated by some elements $\left\{b_{i j}\right\}_{1 \leq j \leq n}$ with $b_{i j} \in H^{2}\left(F_{i} ; Z_{2}\right)$ and $b_{i j}^{2}=0$ for all $j$. In particular, $H^{*}\left(F_{i} ; Z_{2}\right)$ contains a subring which is isomorphic to $H^{*}\left(\left(S^{2}\right)^{k / 2} ; Z_{2}\right)$.

Proof. First the spectral sequence which converges to $H^{*}\left(M_{G} ; Z_{2}\right)$ with $E_{2}^{p, q}=$ $H^{p}\left(R P^{\infty} ; H^{q}\left(M ; Z_{2}\right)\right)$ collapses. By [3, Theorem 1.6, p374], $M$ is totally nonhomologous to zero with coefficient in $Z_{2}$. Let $\left\{c_{t}\right\}_{t=1,2, \ldots, n}$ be elements of $H^{2}\left(M_{G} ; Z_{2}\right)$ such that their restrictions on $M$ form a basis of the $Z_{2}$ vector space $H^{2}\left(M ; Z_{2}\right)$. Let $j_{1}:\left(F_{i}\right)_{G}=R P^{\infty} \times F_{i} \rightarrow M_{G}$ be the inclusion. Then $j_{1}^{*}: H^{k}\left(M_{G} ; Z_{2}\right) \rightarrow$ $H^{k}\left(\left(F_{i}\right)_{G} ; Z_{2}\right)$ is a surjection for $k>2 n$ ([3, Theorem 1.5, p374]). Note that $H^{*}\left(\left(F_{i}\right)_{G} ; Z_{2}\right) \approx H^{*}\left(R P^{\infty} ; Z_{2}\right) \otimes H^{*}\left(F_{i} ; Z_{2}\right)$. Let

$$
j_{1}^{*}\left(c_{t}\right)=a \otimes b_{i t 1}+1 \otimes b_{i t 2}+a^{2} \otimes 1
$$

where $b_{i t j} \in H^{j}\left(F_{i} ; Z_{2}\right)$ for $j=1,2$ and $a \in H^{1}\left(R P^{\infty} ; Z_{2}\right)$ is a generator. Since $j_{1}^{*}$ is onto in high degrees, $H^{*}\left(F_{i} ; Z_{2}\right)$ is algebraically generated by the set $\left\{1, b_{i t 1}, b_{i t 2}\right\}_{t=1,2, \ldots, n}$.

Next by the assumed condition, $S q^{1}\left(c_{t}\right) \in p^{*} H^{3}\left(R P^{\infty} ; Z_{2}\right)$; thus $j_{1}^{*} S q^{1}\left(c_{t}\right) \in$ $p^{*} H^{*}\left(R P^{\infty} ; Z_{2}\right)$. We claim $b_{i t 1}=0$. Otherwise,

$$
\begin{aligned}
j_{1}^{*} S q^{1}\left(c_{t}\right) & =S q^{1} j_{1}^{*}\left(c_{t}\right) \\
& =S q^{1}\left(1 \otimes b_{i t 2}+a \otimes b_{i t 1}+a^{2} \otimes 1\right) \\
& =1 \otimes S q^{1} b_{i t 2}+a^{2} \otimes b_{i t 1}+a \otimes\left(b_{i t 1}\right)^{2} \\
& \notin p^{*} H^{*}\left(R P^{\infty} ; Z_{2}\right) .
\end{aligned}
$$

This is a contradiction. Now we claim $\left(b_{i t 2}\right)^{2}=0$ for each $t$. Let $j: M \rightarrow M_{G}$, 
$\tilde{j}: F_{i} \rightarrow\left(F_{i}\right)_{G}$ and $\tilde{j}_{1}: F_{i} \rightarrow M$ be inclusions. Then the diagram

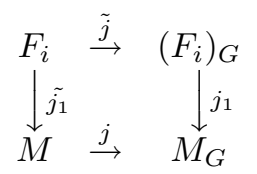

commutes. Thus we have $b_{i t 2}=\left(j_{1} \tilde{j}\right)^{*}\left(c_{t}\right)=\left(\tilde{j_{1}}\right)^{*} j^{*}\left(c_{t}\right)$ and $b_{i t 2}^{2}=\left(\tilde{j_{1}}\right)^{*}\left(j^{*}\left(c_{t}\right)\right)^{2}=$ $\left(\tilde{j_{1}}\right)^{*}(0)=0$.

Note that by Theorem 1.2 (ii), $F$ is of constant dimension. Let $k$ be the dimension of $F$; then the generator of $H^{k}\left(F_{i} ; Z_{2}\right)$ must be a product of some $b_{i t 2}$ 's. Consequently, $k$ must be even and $H^{*}\left(F_{i} ; Z_{2}\right)$ has a subring which is isomorphic to $H^{*}\left(\left(S^{2}\right)^{k / 2} ; Z_{2}\right)$. This together with the equation $\sum_{j \geq 0} \operatorname{rank} H^{j}\left(M ; Z_{2}\right)=2^{n}$ ([3, Theorem 1.6, p374]) shows the number of the components of $F$ is at most $2^{n-k / 2}$.

Proof of Theorem 1.3. First we prove the statement (i). By the Lefschetz fixed point theorem, $F$ is nonempty. By Theorem 1.2 (ii), $F$ is of constant dimension. Note that $S q^{1}=\rho \beta$, where $\rho$ and $\beta$ fit into the Bockstein exact sequence

$$
\rightarrow H^{2}\left(M_{G} ; Z_{2}\right) \stackrel{\beta}{\rightarrow} H^{3}\left(M_{G} ; Z\right) \stackrel{2}{\rightarrow} H^{3}\left(M_{G} ; Z\right) \stackrel{\rho}{\rightarrow} H^{3}\left(M_{G} ; Z_{2}\right) \rightarrow .
$$

We claim $H^{\text {odd }}\left(M_{G} ; Z\right)=0$. Indeed, the spectral sequence which converges to $H^{*}\left(M_{G} ; Z\right)$ with $E_{2}^{p, q}=H^{p}\left(R P^{\infty} ; H^{q}(M ; Z)\right)$ collapses. Since

$$
H^{q}(M ; Z)= \begin{cases}0 & \text { if } q \text { is odd, } \\ \text { free abelian } & \text { if } q \text { is even, }\end{cases}
$$

and $H^{\text {odd }}\left(R P^{\infty} ; Z\right)=0, H^{\text {odd }}\left(M_{G} ; Z\right)$ must be trivial. This implies $\beta=0$ and $S q^{1}\left(c_{i}\right)=\rho \beta\left(c_{i}\right)=0$, where $\left\{c_{i}\right\}$ are as those in the proof of Proposition 2.4. Finally, $\tau$ induces the trivial action on $H^{*}(M ; Z)$ implies the triviality of the induced $Z_{2}$ action on $H^{*}\left(M ; Z_{2}\right)$. So (i) follows from Proposition 2.4.

Next we consider (ii). Similarly to the proof of Proposition 2.4, let $\left\{c_{t}\right\}_{1 \leq j \leq n}$ be the elements of $H^{1}\left(M_{G} ; Z_{2}\right)$ such that $\left\{j^{*}\left(c_{t}\right)\right\}$ is a basis of the $Z_{2}$ vector space $H^{1}\left(M^{n} ; Z_{2}\right)$, and let

$$
j_{1}^{*}\left(c_{t}\right)=1 \otimes b_{i t}+a \otimes 1, \quad a \in H^{1}\left(R P^{\infty} ; Z_{2}\right), b_{i t} \in H^{1}\left(F_{i} ; Z_{2}\right) .
$$

Here we use the notation of Proposition 2.4. Then $\left\{1, b_{1 t}, b_{2 t}, \ldots, b_{n t}\right\}$ generate algebraically the $Z_{2}$ algebra $H^{*}\left(F_{i} ; Z_{2}\right)$, and just as in the proof of Proposition 2.4, we have $\left(b_{i t}\right)^{2}=0$, and (ii) follows as (i).

\section{ACKNOWLEDGMENT}

I would like to thank the referee for many helpful comments, particularly for pointing out to me that we should assume that $H^{*}\left(M^{n} ; Z\right)$ has no 2-torsions in Theorem 1.1, and that the structure of $H^{*}\left(F_{i} ; Z_{2}\right)$ in Theorem 1.3 may have something more than $H^{*}\left(\left(S^{2}\right)^{k / 2} ; Z_{2}\right)$ or $H^{*}\left(\left(S^{1}\right)^{k} ; Z_{2}\right)$. Special thanks to Professors D. M. Davis, Zhende Wu, Zaisi Zuo, and Zhongze Liu, from whom I learned my algebraic topology. I am also grateful to Lehigh University for the financial support while this work was performed. 


\section{REFERENCES}

[1] M. F. Atiyah, K-Theory, Benjamin, New York, 1967. MR 36:7130

[2] A. Borel and Hirzebruch, On the characteristic classes of the homogeneous spaces, Amer. J. Math., Vol. 80 (1958), 458-538. MR 21:1586

[3] G. E. Bredon, Introduction to compact transformation groups, Academic Press, New York and London (1972). MR 54:1265

[4] J. W. Milnor and Stashelff, Characteristic classes, Annals of Math. Studies, No.76(1974). MR 55:13428

[5] Huajian Yang, Wu Zhende and Liu Zongze, Involution number sequence and its applications (II), Science in China(Ser.A), 35(1992), pp819-825. MR 94a:57050

[6] Huajian Yang, Involution number sequence and its applications (I), Science in China (Ser. A), 34(1991), pp541-545.

Department of Mathematics, Lehigh University, Bethlehem, Pennsylvania 18015

E-mail address: hy02@lehigh.edu

Department of Mathematics, South China Normal University, Guangzhou, People's Republic of China, 510631 